\title{
Discrete-time Lyapunov based small-gain theorem for parameterized interconnected ISS systems
}

\author{
Dina Shona Laila and Dragan Nešić \\ Electrical and Electronic Engineering Department, \\ The University of Melbourne, Parkville, 3010, Victoria, Australia
}

\begin{abstract}
Input-to-state stability (ISS) of a feedback interconnection of two discrete-time ISS systems satisfying an appropriate small gain condition is investigated via the Lyapunov method. In particular, an ISS Lyapunov function for the overall system is constructed from the ISS Lyapunov functions of the two subsystems. We consider parameterized families of discrete-time systems that naturally arise when an approximate discrete-time model is used in controller design for a sampled-data system.
\end{abstract}

Keywords: Discrete-time; Small gain; Input-to-state stability; Lyapunov method; Nonlinear.

\section{Introduction}

The small gain theorem is one of the most important tools in robustness analysis and controller design for nonlinear control systems. A particularly useful version of the small gain theorem for nonlinear continuous-time systems was proved in [3] by Jiang et al. and it is based on the input-to-state stability (ISS) property introduced by Sontag in [12] (see also [13]). A range of related result for continuous-time systems can be found in $[2,11,14,15]$ and for nonlinear discrete-time systems in [5]. All of the above results rely on trajectory based proofs of the small gain theorem and they do not construct a Lyapunov function for the overall interconnected system. The first partial construction of a Lyapunov function for the feedback connection of two continuous-time ISS systems satisfying a small-gain condition that we are aware of was proposed in [4].

It is the main purpose of this paper to present a discrete-time version of the results in [4]. Indeed, we present a partial construction of an ISS Lyapunov function from the ISS Lyapunov functions of two interconnected discrete-time ISS systems satisfying a small-gain condition. While the constructed Lyapunov function in the discrete-time case has the same form as the one constructed in [4] for continuous-time systems, the proofs of the two results are significantly different.

Our main result is a useful tool for a range of nonlinear discrete-time control problems. In particular, the constructed Lyapunov function can be used together with results in [7, 9] to design ISS controllers for nonlinear sampled-data systems via their approximate discrete-time plant models. We also remark that our main result is closely related to results on changes of supply rates for ISS discrete-time systems investigated in [10] and for IOSS discrete-time systems investigated in [6] and it can be regarded as an appropriate generalization of the results in [10]. 


\section{Preliminaries}

The set of real numbers is denoted by $\mathbb{R}$. A function $\gamma: \mathbb{R}_{\geq 0} \rightarrow \mathbb{R}_{\geq 0}$ is of class $\mathcal{K}$ if it is continuous, strictly increasing and zero at zero; it is of class $\mathcal{K}_{\infty}$ if it is of class $\mathcal{K}$ and unbounded. Functions of class $\mathcal{K}_{\infty}$ are invertible. We say that a function $q: \mathbb{R}_{\geq 0} \rightarrow \mathbb{R}_{>0}$ is positive if it is continuous and $q(s)>0$ for all $s \geq 0$. A function $q: \mathbb{R}_{\geq 0} \rightarrow \mathbb{R}_{\geq 0}$ is positive definite if it is continuous, $q(0)=0$ and $q(s)>0$ for all $s>0$. A function $\alpha: \mathbb{R}_{\geq 0} \rightarrow \mathbb{R}_{>0}$ is of class $\mathcal{L}$ if it is positive and $\alpha(s)$ is strictly decreasing to zero as $s \rightarrow \infty$. Given two functions $\alpha(\cdot)$ and $\gamma(\cdot)$, we denote their composition and multiplication respectively as $\alpha \circ \gamma(\cdot)$ and $\alpha(\cdot) \cdot \gamma(\cdot)$. Identity function is denoted by $\operatorname{Id}$, that is $\operatorname{Id}(s):=s$.

Consider a family of parameterized discrete-time systems

$$
x(k+1)=F_{T}(x(k), u(k)) .
$$

where $x \in \mathbb{R}^{n}$ and $u \in \mathbb{R}^{m}$ are respectively the state and input of the system. It is assumed that $F_{T}$ is well defined on arbitrarily large compact sets for sufficiently small $T$, where $T>0$ is the sampling period, which parameterizes the system and can be arbitrarily assigned. Parameterized discrete-time systems (1) commonly arise when an approximate discrete-time model is used for designing a digital controller for a nonlinear sampled-data system (see [7,9]). For instance, if we use the Euler model of $\dot{x}=f(x, u)$ for controller design then we have $F_{T}(x, u):=x+T f(x, u)$. Non-parameterized discrete-time systems are a special case of (1) when $T$ is constant (for instance $T=1$ ). We use the following definition.

Definition 2.1 The system (1) is semiglobally practically input-to-state stable (SP-ISS) w.r.t. input u if there exist functions $\underline{\alpha}, \bar{\alpha} \in \mathcal{K}_{\infty}$, a positive definite function $\alpha$ and $\gamma \in \mathcal{K}$, and for any strictly positive real numbers $\Delta_{x}, \Delta_{u}, \nu$ and $\tilde{\nu}$ there exists $T^{*}>0$ such that for all $T \in\left(0, T^{*}\right)$ there exists a continuous function $V_{T}: \mathbb{R}^{n} \rightarrow \mathbb{R}_{\geq 0}$ such that for all $|x| \leq \Delta_{x},|u| \leq \Delta_{u}$ and $T \in\left(0, T^{*}\right)$ the following holds:

$$
\begin{gathered}
\underline{\alpha}(|x|) \leq V_{T}(x) \leq \bar{\alpha}(|x|), \\
V_{T}(x) \geq \gamma(|u|)+\nu \Rightarrow V_{T}\left(F_{T}\right)-V_{T}(x) \leq-T \alpha\left(V_{T}(x)\right), \\
V_{T}\left(F_{T}\right) \leq V_{T}(x)+\tilde{\nu} .
\end{gathered}
$$

The function $V_{T}$ is called a SP-ISS Lyapunov function for the system (1).

Definition 2.2 (Lipschitz uniform in small T) A family of functions $V_{T}: \mathbb{R}^{n} \rightarrow \mathbb{R}_{\geq 0}$ is Lipschitz uniformly in small $T$ if given any $\Delta_{x}>0$ there exists $T^{*}>0$ such that for all $T \in\left(0, T^{*}\right)$ and $\max \{|x|,|y|\} \leq \Delta_{x}$ the following holds:

$$
\left|V_{T}(x)-V_{T}(y)\right| \leq L|x-y|
$$

Remark 2.1 We note that for continuous-time systems, if $\Delta_{x}>\underline{\alpha}^{-1}\left(\gamma\left(\Delta_{u}\right)+\nu\right)$ then the condition (4) is not needed in the definition of SP-ISS and a condition that corresponds to (3) is enough to guarantee an appropriate ISS bound on the trajectories of the system. However, for discrete-time systems, the condition (3) alone is not enough to guarantee even the boundedness of the trajectories of the system no 
matter how large $\Delta_{x}$ is compared to $\Delta_{u}$ and $\nu$. This is illustrated by the system $x(k+1)=F_{T}(x(k))$ where $F_{T}(\cdot)$ is any continuous function satisfying (the example is taken from [8])

$$
F_{T}(x)= \begin{cases}2 \Delta_{x} & |x| \leq \nu / 2 \\ 2|x| & |x| \geq 2 \Delta_{x} \\ 0 & \nu \leq|x| \leq \Delta_{x}\end{cases}
$$

and $\Delta_{x}$ and $\nu$ are arbitrarily positive real numbers. With, for example, $V_{T}(x)=|x|$, we have for all $|x| \leq \Delta_{x}$ that

$$
|x| \geq \nu \Rightarrow \Delta V_{T}:=V_{T}\left(F_{T}(x)\right)-V_{T}(x)=-V_{T}(x) .
$$

Yet, every trajectory grows without bound. Note that the condition (7) gives the right bound on $\Delta V_{T}$ for all $\Delta_{x} \geq|x| \geq \nu$. However, this example shows that some information about $V_{T}\left(F_{T}(x)\right)$ is required even for values of $x$ such that $|x| \leq \nu$ in order to assert a bound on trajectories of the system. Consequently, we have included the condition (4) as a part of SP-ISS characterization in Definition 2.1. We note that the condition (4) is not restrictive and is satisfied in most situations of interest. Example 2.1 illustrates a particular case of this condition.

Example 2.1 Consider a continuous-time nonlinear system $\dot{x}=f(x, u)$ where $f$ is bounded on compact sets. Suppose we use the Euler discrete-time model of the system $x(k+1)=F_{T}(x(k), u(k)):=x(k)+$ $T f(x(k), u(k))$ to analyse its properties. Consider also a Lyapunov function $V_{T}$ that is uniformly (locally) Lipschitz in small $T$. Then, we can write on compact sets:

$$
V_{T}\left(F_{T}\right)=V_{T}(x)+V_{T}(x+T f(x, u))-V_{T}(x) \leq V_{T}(x)+L T|f(x, u)| .
$$

Since there exists $M>0$ so that $|f(x, u)| \leq M$, then given any $\tilde{\nu}>0$ there exists $T^{*}>0$ (we can take $\left.T^{*}=\frac{\tilde{\nu}}{L M}\right)$ so that for all $T \in\left(0, T^{*}\right)$ we have that (4) holds.

Remark 2.2 If instead of (3), we used the following Lyapunov condition in Definition 2.1

$$
\Delta V_{T} \leq-T a(|x|)+T \gamma(|u|)+T \nu, \quad a, \gamma \in \mathcal{K}_{\infty},
$$

then we would not need (4). However, the above given formulation leads to a more complicated statement and proof of our main result and hence we have opted to use the conditions as stated in Definition 2.1. We emphasize that (3) and (4) are equivalent to (9) if an appropriate condition holds. Indeed, it is trivial to see that (9) implies both (3) and (4). The opposite holds if there exists $\sigma \in \mathcal{K}_{\infty}$ such that for any strictly positive $r, \nu$ there exists $T^{*}>0$ such that the following holds

$$
\max _{T \in\left(0, T^{*}\right),|x| \leq \gamma(r),|u| \leq r}\left|\frac{\Delta V_{T}}{T}\right| \leq \sigma(r)+\nu,
$$

and then we can write that for any $\left(\Delta_{x}, \Delta_{u}, \nu\right)$ there exists $T^{*}>0$ such that the following holds:

$$
\Delta V_{T} \leq-T \alpha\left(V_{T}\right)+T \sigma(|u|)+T \nu .
$$

The condition (10) is slightly stronger than (4) but it often holds. 
We note that the condition (9) was used in [9] to provide a framework for design of input-to-state stabilizing controllers for sampled-data systems via their approximate discrete-time models. Hence, results of this paper in cases when the condition (10) holds provide a tool for ISS controller design within the framework of [9]. In Section 4 we present an example which illustrates the importance of the particular definition of SP-ISS that we use when the controller design is based on an approximate discrete-time plant model.

\section{Main result}

In this section we state and prove Theorem 3.1, which is the main result of this paper. Theorem 3.1 is a discrete-time version of the continuous-time result [4]. The statements of both results are similar but the proofs are notably different and the differences are commented on below (see Remark 3.2).

The focus of this paper is a family of parameterized discrete-time interconnected systems

$$
\begin{aligned}
& \Sigma_{1}: x_{1}(k+1)=F_{1 T}\left(x_{1}(k), x_{2}(k), u(k)\right), \\
& \Sigma_{2}: x_{2}(k+1)=F_{2 T}\left(x_{1}(k), x_{2}(k), u(k)\right) .
\end{aligned}
$$

In the sequel we will assume that the subsystem $\Sigma_{1}$ is SP-ISS with respect to inputs $x_{2}$ and $u$ and the subsystem $\Sigma_{2}$ is SP-ISS with respect to inputs $x_{1}$ and $u$. More precisely, we suppose that for $i, j \in\{1,2\}, i \neq j$, there exist functions $\underline{\alpha}_{i}, \bar{\alpha}_{i} \in \mathcal{K}_{\infty}$, positive definite functions $\alpha_{i}$, functions $\gamma_{x_{i}}, \gamma_{u_{i}} \in \mathcal{K}$, and for any strictly positive real numbers $\left(\Delta_{x_{i}}, \Delta_{x_{j}}, \Delta_{u_{i}}, \nu_{i}, \tilde{\nu}_{i}\right)$ there exist $T_{i}^{*}>0$ and for any $T \in\left(0, T_{i}^{*}\right)$ there exist $V_{i T}: \mathbb{R}^{n} \rightarrow \mathbb{R}_{\geq 0}$ such that the following hold for all $T \in\left(0, T_{i}^{*}\right),\left|x_{i}\right| \leq \Delta_{x_{i}},\left|x_{j}\right| \leq \Delta_{x_{j}}$ and $|u| \leq \Delta_{u_{i}}$ :

$$
\begin{gathered}
\underline{\alpha}_{i}\left(\left|x_{i}\right|\right) \leq V_{i T}\left(x_{i}\right) \leq \bar{\alpha}_{i}\left(\left|x_{i}\right|\right), \\
V_{i T}\left(x_{i}\right) \geq \max \left\{\gamma_{x_{i}}\left(V_{j T}\left(x_{j}\right)\right), \gamma_{u_{i}}(|u|)+\nu_{i}\right\} \Rightarrow V_{i T}\left(F_{i T}\right)-V_{i T}\left(x_{i}\right) \leq-T \alpha_{i}\left(V_{i T}\left(x_{i}\right)\right), \\
V_{i T}\left(F_{i T}\right) \leq V_{i T}\left(x_{i}\right)+\tilde{\nu}_{i} .
\end{gathered}
$$

Under the above given conditions and an appropriate small gain condition, we show that the overall system (11) is SP-ISS with respect to the input $u$. Moreover, we construct a SP-ISS Lyapunov function $V_{T}$ for the overall system (11) using the SP-ISS Lyapunov functions $V_{1 T}$ and $V_{2 T}$ of the subsystems $\Sigma_{1}$ and $\Sigma_{2}$. More precisely, we can state the following result.

Theorem 3.1 Consider the family of parameterized discrete-time interconnected system (11). Suppose that the following conditions hold:

A1. The subsystem $\Sigma_{1}$ is SP-ISS with inputs $x_{2}$ and $u$ and SP-ISS Lyapunov function $V_{1 T}$.

A2. The subsystem $\Sigma_{2}$ is SP-ISS with inputs $x_{1}$ and $u$ and SP-ISS Lyapunov function $V_{2 T}$.

A3. There exist $\tau_{1}, \tau_{2} \in \mathcal{K}_{\infty}$ such that $\left(\mathrm{Id}+\tau_{1}\right) \circ \gamma_{x_{1}} \circ\left(\mathrm{Id}+\tau_{2}\right) \circ \gamma_{x_{2}}(s)<s, \quad \forall s>0$.

Then, the system (11) is SP-ISS w.r.t. the input u; moreover, there exists $\rho \in \mathcal{K}_{\infty}$ such that the function

$$
V_{T}\left(x_{1}, x_{2}\right):=\max \left\{V_{1 T}\left(x_{1}\right), \rho\left(V_{2 T}\left(x_{2}\right)\right)\right\},
$$

is SP-ISS Lyapunov function for the system (11). Moreover, if $V_{1 T}, V_{2 T}$ are locally Lipschitz uniformly in small $T$, then $V_{T}$ is locally Lipschitz uniformly in small $T$. 
Proof of Theorem 3.1: Suppose that all conditions of Theorem 3.1 are satisfied. Let $\underline{\alpha}_{1}, \bar{\alpha}_{1}, \alpha_{1}, \gamma_{x_{1}}$, $\gamma_{u_{1}}$ come from conditions A1, and let $\underline{\alpha}_{2}, \bar{\alpha}_{2}, \alpha_{2}, \gamma_{x_{2}}, \gamma_{u_{2}}$ come from condition A2. Let $\tau_{1}, \tau_{2} \in \mathcal{K}_{\infty}$ come from condition A3. Note that without loss of generality we can assume that $\left(\operatorname{Id}-\alpha_{i}\right), i=1,2$ are positive definite. For simplicity of notation we introduce $\tilde{\gamma}_{x_{1}}(s):=\left(\operatorname{Id}+\tau_{1}\right) \circ \gamma_{x_{1}}, \quad \tilde{\gamma}_{x_{2}}(s):=\left(\operatorname{Id}+\tau_{2}\right) \circ \gamma_{x_{2}}$. Similar to [4] we denote $b:=\lim _{r \rightarrow \infty} \tilde{\gamma}_{x_{2}}(r)$ and since $\tilde{\gamma}_{x_{2}} \in \mathcal{K}$, then $\tilde{\gamma}_{x_{2}}^{-1}$ is defined on $[0, b), \tilde{\gamma}_{x_{2}}^{-1}(r) \rightarrow \infty$ as $r \rightarrow b^{-}$and from A3 we have that

$$
\tilde{\gamma}_{x_{1}}(r)<\tilde{\gamma}_{x_{2}}^{-1}(r), \forall r \in(0, b)
$$

Let $\hat{\gamma}_{x} \in \mathcal{K}_{\infty}$ be such that

- $\hat{\gamma}_{x}(r) \leq \tilde{\gamma}_{x_{2}}^{-1}(r)$ for all $r \in[0, b)$;

- $\tilde{\gamma}_{x_{1}}(r)<\hat{\gamma}_{x}(r)$ for all $r>0$.

(if $\tilde{\gamma}_{x_{2}} \in \mathcal{K}_{\infty}$, then we can take $\hat{\gamma}_{x}(r)=\tilde{\gamma}_{x_{2}}^{-1}(r)$ ). Let $\rho \in \mathcal{K}_{\infty}$ come from Lemma 6.1 such that

$$
\tilde{\gamma}_{x_{1}}(r)<\rho(r)<\hat{\gamma}_{x}(r), \quad \forall r>0 .
$$

Denote $\tilde{q}(r):=\frac{d \rho}{d r}(r)$, where $\tilde{q}$ is a positive function. Let $V_{T}$ be defined as:

$$
V_{T}\left(x_{1}, x_{2}\right):=\max \left\{V_{1 T}\left(x_{1}\right), \rho\left(V_{2 T}\left(x_{2}\right)\right)\right\} .
$$

We use the notation $x:=\left(x_{1}^{T} x_{2}^{T}\right)^{T}, F_{T}:=\left(F_{1 T}^{T} F_{2 T}^{T}\right)^{T}$ and the norm $|x|:=\left|x_{1}\right|+\left|x_{2}\right|$. We show that the interconnected system (11) is SP-ISS with input $u$ by proving that $V_{T}$ is a SP-ISS Lyapunov function for the system.

Let arbitrary strictly positive real numbers $\left(\Delta_{x}, \Delta_{u}, \nu, \tilde{\nu}\right)$ be given. Let $\Delta_{x_{1}}=\Delta_{x_{2}}=\Delta_{x}$ and $\Delta_{u_{1}}=\Delta_{u_{2}}=\Delta_{u}$. Let $\varepsilon_{1}, \varepsilon_{2} \in \mathcal{K}_{\infty}$ be arbitrary functions such that $\left(\mathrm{Id}-\varepsilon_{i}\right)$ are positive definite functions for $i=1,2$. Let $\nu_{1}$ be such that

$$
\max \left\{\nu_{1}, \max _{s \in\left[0, \Delta_{u}\right]}\left[\varepsilon_{1}^{-1}\left(\gamma_{u_{1}}(s)+\nu_{1}\right)-\varepsilon_{1}^{-1} \circ \gamma_{u_{1}}(s)\right]\right\} \leq \nu
$$

and let $\nu_{2}$ be such that

$$
\max \left\{\max _{s \in\left[0, \Delta_{u}\right]}\left[\rho\left(\gamma_{u_{2}}(s)+\nu_{2}\right)-\rho \circ \gamma_{u_{2}}(s)\right], \max _{s \in\left[0, \Delta_{u}\right]}\left[\varepsilon_{2}^{-1} \circ \rho\left(\gamma_{u_{2}}(s)+\nu_{2}\right)-\varepsilon_{2}^{-1} \circ \rho \circ \gamma_{u_{2}}(s)\right]\right\} \leq \nu .
$$

Let $\tilde{\nu}_{1}>0$ and $\tilde{\nu}_{2}>0$ be such that

$$
\begin{gathered}
\max \left\{\tilde{\nu}_{1}, \max _{s \in\left[0, \bar{\alpha}_{2}\left(\Delta_{x_{2}}\right)\right]}\left[\rho\left(s+\tilde{\nu}_{2}\right)-\rho(s)\right]\right\} \leq \tilde{\nu}, \\
\left(\operatorname{Id}+\tau_{1}^{-1}\right)\left(\tilde{\nu}_{1}\right) \leq \frac{\nu_{1}}{2}, \quad\left(\operatorname{Id}+\tau_{2}^{-1}\right)\left(\tilde{\nu}_{2}\right) \leq \frac{\nu_{2}}{2} .
\end{gathered}
$$

Let $\left(\Delta_{x_{1}}, \Delta_{x_{2}}, \Delta_{u_{1}}, \frac{\nu_{1}}{2}, \tilde{\nu}_{1}\right)$ determine $T_{1}^{*}>0$ via the condition A1. Let $\left(\Delta_{x_{1}}, \Delta_{x_{2}}, \Delta_{u_{2}}, \frac{\nu_{2}}{2}, \tilde{\nu}_{2}\right)$ determine $T_{2}^{*}>0$ via the condition A2. Let $T^{*}:=\min \left\{1, T_{1}^{*}, T_{2}^{*}\right\}$. In the rest of the proof we assume that $|x| \leq \Delta_{x}$, $|u| \leq \Delta_{u}$ and $T \in\left(0, T^{*}\right)$.

First note that $\tilde{\gamma}_{x_{1}}(s) \geq \gamma_{x_{1}}(s), \tilde{\gamma}_{x_{2}}(s) \geq \gamma_{x_{2}}(s)$ for all $s \geq 0$. Conditions A1 and A2 imply that:

$$
\begin{array}{cl}
V_{1 T}\left(x_{1}\right) \geq \max \left\{\gamma_{x_{1}}\left(V_{2 T}\left(x_{2}\right)\right), \gamma_{u_{1}}(|u|)+\nu_{1} / 2\right\} & \Rightarrow V_{1 T}\left(F_{1 T}\right)-V_{1 T}\left(x_{1}\right) \leq-T \alpha_{1}\left(V_{1 T}\left(x_{1}\right)\right) \\
V_{2 T}\left(x_{2}\right) \geq \max \left\{\gamma_{x_{2}}\left(V_{1 T}\left(x_{1}\right)\right), \gamma_{u_{2}}(|u|)+\nu_{2} / 2\right\} & \Rightarrow \quad V_{2 T}\left(F_{2 T}\right)-V_{2 T}\left(x_{2}\right) \leq-T \alpha_{2}\left(V_{2 T}\left(x_{2}\right)\right) \\
V_{1 T}\left(F_{1 T}\right) \leq V_{1 T}\left(x_{1}\right)+\tilde{\nu}_{1}, & V_{2 T}\left(F_{2 T}\right) \leq V_{2 T}\left(x_{2}\right)+\tilde{\nu}_{2} .
\end{array}
$$


Moreover, using respectively A1 and A2 and our choice of $\nu_{i}, \tilde{\nu}_{i}, i=1,2$ we can write respectively

$$
\begin{aligned}
& V_{1 T}\left(F_{1 T}\right) \leq \max \left\{\left(\operatorname{Id}-T \alpha_{1}\right)\left(V_{1 T}\left(x_{1}\right)\right), \tilde{\gamma}_{x_{1}}\left(V_{2 T}\left(x_{2}\right)\right), \gamma_{u_{1}}(|u|)+\nu_{1}\right\}, \\
& V_{2 T}\left(F_{2 T}\right) \leq \max \left\{\left(\operatorname{Id}-T \alpha_{2}\right)\left(V_{2 T}\left(x_{2}\right)\right), \tilde{\gamma}_{x_{2}}\left(V_{1 T}\left(x_{1}\right)\right), \gamma_{u_{2}}(|u|)+\nu_{2}\right\} .
\end{aligned}
$$

We only prove (26) and the proof of (27) is omitted since it follows the same steps. Note that if $V_{1 T}\left(x_{1}\right) \geq \max \left\{\gamma_{x_{1}}\left(V_{2 T}\left(x_{2}\right)\right), \gamma_{u_{1}}(|u|)+\nu_{1} / 2\right\}$, then from (23) we can write that:

$$
V_{1 T}\left(F_{1 T}\right) \leq\left(\operatorname{Id}-T \alpha_{1}\right)\left(V_{1 T}\left(x_{1}\right)\right) .
$$

On the other hand, if $V_{1 T}\left(x_{1}\right) \leq \max \left\{\gamma_{x_{1}}\left(V_{2 T}\left(x_{2}\right)\right), \gamma_{u_{1}}(|u|)+\nu_{1} / 2\right\}$, then from (25) we have

$$
V_{1 T}\left(F_{1 T}\right) \leq V_{1 T}\left(x_{1}\right)+\tilde{\nu}_{1} \leq \max \left\{\gamma_{x_{1}}\left(V_{2 T}\left(x_{2}\right)\right)+\tilde{\nu}_{1}, \gamma_{u_{1}}(|u|)+\nu_{1} / 2+\tilde{\nu}_{1}\right\}
$$

By considering two sub-cases $\tau_{1} \circ \gamma_{x_{1}}\left(V_{2 T}\left(x_{2}\right)\right) \geq \tilde{\nu}_{1}$ and $\tau_{1} \circ \gamma_{x_{1}}\left(V_{2 T}\left(x_{2}\right)\right) \leq \tilde{\nu}_{1}$, and from definition of $\nu_{1}$ and $\tilde{\nu}_{1}$ we can write that

$$
\begin{aligned}
V_{1 T}\left(F_{1 T}\right) & \leq \max \left\{\left(\operatorname{Id}+\tau_{1}\right) \circ \gamma_{x_{1}}\left(V_{2 T}\left(x_{2}\right)\right),\left(\operatorname{Id}+\tau_{1}^{-1}\right)\left(\tilde{\nu}_{1}\right), \gamma_{u_{1}}(|u|)+\nu_{1} / 2+\tilde{\nu}_{1}\right\} \\
& \leq \max \left\{\tilde{\gamma}_{x_{1}}\left(V_{2 T}\left(x_{2}\right)\right),\left(\operatorname{Id}+\tau_{1}^{-1}\right)\left(\tilde{\nu}_{1}\right), \gamma_{u_{1}}(|u|)+\nu_{1} / 2+\nu_{1} / 2\right\} \\
& \leq \max \left\{\tilde{\gamma}_{x_{1}}\left(V_{2 T}\left(x_{2}\right)\right), \nu_{1} / 2, \gamma_{u_{1}}(|u|)+\nu_{1}\right\}=\max \left\{\tilde{\gamma}_{x_{1}}\left(V_{2 T}\left(x_{2}\right)\right), \gamma_{u_{1}}(|u|)+\nu_{1}\right\}
\end{aligned}
$$

and (28), (29) complete the proof of (26). We assume in the sequel that (23)-(27) hold.

We have that $\frac{1}{2} r_{1}+\frac{1}{2} \rho\left(r_{2}\right) \leq \max \left\{r_{1}, \rho\left(r_{2}\right)\right\} \leq r_{1}+\rho\left(r_{2}\right)$, for any $\rho \in \mathcal{K}_{\infty}, r_{1} \geq 0, r_{2} \geq 0$, and that for any $\alpha_{1}, \alpha_{2} \in \mathcal{K}$, there exist $\underline{\alpha}, \bar{\alpha} \in \mathcal{K}$ such that $\underline{\alpha}\left(s_{1}+s_{2}\right) \leq \alpha_{1}\left(s_{1}\right)+\alpha_{2}\left(s_{2}\right) \leq \bar{\alpha}\left(s_{1}+s_{2}\right), \forall s_{1} \geq$ $0, s_{2} \geq 0$, where we can take $\underline{\alpha}(s):=\min \left\{\alpha_{1}\left(\frac{s}{2}\right), \alpha_{2}\left(\frac{s}{2}\right)\right\}$ and $\bar{\alpha}(s):=2 \max \left\{\alpha_{1}(s), \alpha_{2}(s)\right\}$. Using the definition of $V_{T}$, we have that $V_{T}$ satisfies (2) with $\underline{\alpha}(s):=\min \left\{1 / 2 \underline{\alpha}_{1}(s / 2), 1 / 2 \rho \circ \underline{\alpha}_{2}(s / 2)\right\}$ and $\bar{\alpha}(|x|):=2 \max \left\{\bar{\alpha}_{1}(s), \rho \circ \bar{\alpha}_{2}(s)\right\}$. Moreover, from the definition of $V_{T}$ and (21) we have that

$$
\begin{aligned}
V_{T}\left(F_{T}\right) & =\max \left\{V_{1 T}\left(F_{1 T}\right), \rho\left(V_{2 T}\left(F_{2 T}\right)\right\} \leq \max \left\{V_{1 T}\left(x_{1}\right)+\tilde{\nu}_{1}, \rho\left(V_{2 T}\left(x_{2}\right)+\tilde{\nu}_{2}\right)\right\}\right. \\
& \leq \max \left\{V_{1 T}\left(x_{1}\right), \rho\left(V_{2 T}\left(x_{2}\right)\right)\right\}+\tilde{\nu}=V_{T}(x)+\tilde{\nu}
\end{aligned}
$$

which proves that (4) holds.

To show that $V_{T}$ satisfies (3), we consider the following four cases:

Case 1: $V_{1 T}\left(x_{1}\right) \geq \rho\left(V_{2 T}\left(x_{2}\right)\right)$ and $V_{1 T}\left(F_{1 T}\right) \geq \rho\left(V_{2 T}\left(F_{2 T}\right)\right)$. It holds that

$$
\Delta V_{T}:=V_{T}\left(F_{T}\right)-V_{T}(x)=V_{1 T}\left(F_{1 T}\right)-V_{1 T}\left(x_{1}\right) .
$$

Conditions $V_{1 T}\left(x_{1}\right) \geq \rho\left(V_{2 T}\left(x_{2}\right)\right)$ and $\gamma_{x_{1}}(r) \leq \tilde{\gamma}_{x_{1}}(r)<\rho(r)$ for all $r>0$ imply $V_{1 T}\left(x_{1}\right)>\gamma_{x_{1}}\left(V_{2 T}\left(x_{2}\right)\right)$. Hence, from (23) it holds that if $V_{1 T}\left(x_{1}\right) \geq \gamma_{u_{1}}(|u|)+\nu_{1}$ then we have

$$
V_{1 T}\left(F_{1 T}\right)-V_{1 T}\left(x_{1}\right) \leq-T \alpha_{1}\left(V_{1 T}\left(x_{1}\right)\right) .
$$

Since $V_{T}(x)=V_{1 T}\left(x_{1}\right), \nu \geq \nu_{1}$ and $\varepsilon_{1}^{-1}>\mathrm{Id}$ we have

$$
V_{T}(x) \geq \varepsilon_{1}^{-1} \circ \gamma_{u_{1}}(|u|)+\nu \geq \gamma_{u_{1}}(|u|)+\nu \Rightarrow \Delta V_{T} \leq-T \alpha_{1}\left(V_{T}(x)\right) .
$$


Case 2: $V_{1 T}\left(x_{1}\right)<\rho\left(V_{2 T}\left(x_{2}\right)\right)$ and $V_{1 T}\left(F_{1 T}\right)<\rho\left(V_{2 T}\left(F_{2 T}\right)\right)$. It holds that

$$
\Delta V_{T}=\rho\left(V_{2 T}\left(F_{2 T}\right)\right)-\rho\left(V_{2 T}\left(x_{2}\right)\right) .
$$

Conditions $V_{1 T}\left(x_{1}\right)<\rho\left(V_{2 T}\left(x_{2}\right)\right)$ and $\rho^{-1}(r)>\tilde{\gamma}_{x_{2}}(r) \geq \gamma_{x_{2}}(r), \forall r>0$ imply $V_{2 T}\left(x_{2}\right)>\gamma_{x_{2}}\left(V_{1 T}\left(x_{1}\right)\right)$. Hence, from (24) it holds that if $V_{2 T}\left(x_{2}\right) \geq \gamma_{u_{2}}(|u|)+\nu_{2}$, we have that

$$
\Delta V_{2 T}=V_{2 T}\left(F_{2 T}\right)-V_{2 T}\left(x_{2}\right) \leq-T \alpha_{2}\left(V_{2 T}\left(x_{2}\right)\right) \Rightarrow V_{2 T}\left(F_{2 T}\right) \leq\left(\operatorname{Id}-T \alpha_{2}\right)\left(V_{2 T}\left(x_{2}\right)\right) .
$$

Then using the Mean Value Theorem and the construction of $\rho$ via Lemma 6.1, we have that

$$
\begin{aligned}
\Delta V_{T} & =\rho\left(V_{2 T}\left(F_{2 T}\right)\right)-\rho\left(V_{2 T}\left(x_{2}\right)\right) \\
& \leq \rho \circ\left(\operatorname{Id}-T \alpha_{2}\right)\left(V_{2 T}\left(x_{2}\right)\right)-\rho\left(V_{2 T}\left(x_{2}\right)\right)=-T \tilde{q}\left(V_{2 T}^{\star}\right) \cdot \alpha_{2}\left(V_{2 T}\left(x_{2}\right)\right),
\end{aligned}
$$

with $V_{2 T}^{\star} \in\left[\left(\operatorname{Id}-\alpha_{2}\right)\left(V_{2 T}\left(x_{2}\right)\right), V_{2 T}\left(x_{2}\right)\right]$ (since $T<1$ ) and $\tilde{q}$ is a positive function. Let $\tilde{q}$ generate via Lemma 6.2 the functions $q_{1} \in \mathcal{K}_{\infty}$ and $q_{2} \in \mathcal{L}$. We use the fact that $V_{T}(x)=\rho\left(V_{2 T}\left(x_{2}\right)\right)$ to write that

$$
\begin{aligned}
\Delta V_{T} & \leq-T \tilde{q}\left(V_{2 T}^{\star}\right) \cdot \alpha_{2}\left(V_{2 T}\left(x_{2}\right)\right) \\
& \leq-T q_{1}\left(V_{2 T}^{\star}\right) \cdot q_{2}\left(V_{2 T}^{\star}\right) \cdot \alpha_{2}\left(V_{2 T}\left(x_{2}\right)\right) \\
& \leq-T q_{1} \circ\left(\operatorname{Id}-\alpha_{2}\right)\left(V_{2 T}\left(x_{2}\right)\right) \cdot q_{2}\left(V_{2 T}\left(x_{2}\right)\right) \cdot \alpha_{2}\left(V_{2 T}\left(x_{2}\right)\right) \\
& =:-T a_{2 a}\left(V_{2 T}\left(x_{2}\right)\right)=-T a_{2 a} \circ \rho^{-1}\left(V_{T}(x)\right)=-T a_{2}\left(V_{T}(x)\right) .
\end{aligned}
$$

Since $V_{T}(x)=\rho\left(V_{2 T}\left(x_{2}\right)\right), \varepsilon_{2}^{-1}>\operatorname{Id}$ and by $(20)$, we have

$$
V_{T}(x) \geq \varepsilon_{2}^{-1} \circ \rho \circ \gamma_{u_{2}}(|u|)+\nu \geq \rho \circ \gamma_{u_{2}}(|u|)+\nu \Rightarrow \Delta V_{T} \leq-T a_{2}\left(V_{T}(x)\right) .
$$

Case 3: $V_{1 T}\left(x_{1}\right)<\rho\left(V_{2 T}\left(x_{2}\right)\right)$ and $V_{1 T}\left(F_{1 T}\right) \geq \rho\left(V_{2 T}\left(F_{2 T}\right)\right)$. Using (26) it holds that

$$
\begin{aligned}
\Delta V_{T} & =V_{1 T}\left(F_{1 T}\right)-\rho\left(V_{2 T}\left(x_{2}\right)\right) \\
& \leq \max \left\{\left(\operatorname{Id}-T \alpha_{1}\right)\left(V_{1 T}\left(x_{1}\right)\right), \tilde{\gamma}_{x_{1}}\left(V_{2 T}\left(x_{2}\right)\right), \gamma_{u_{1}}(|u|)+\nu_{1}\right\}-\rho\left(V_{2 T}\left(x_{2}\right)\right) .
\end{aligned}
$$

We now over bound each of the terms in (37). First, by using (61) from Lemma 6.3 with $V_{1 T}\left(x_{1}\right)=s$ and $V_{2 T}\left(x_{2}\right)=r$, we obtain

$$
\left(\operatorname{Id}-T \alpha_{1}\right)\left(V_{1 T}\left(x_{1}\right) \leq-T a_{3 a} \circ \rho\left(V_{2 T}\left(x_{2}\right)\right)=-T a_{3 a}\left(V_{T}(x)\right) .\right.
$$

Next, since $\tilde{\gamma}_{x_{1}}(r)<\rho(r), \forall r>0$, the function $a_{3 b}(r):=\rho(r)-\tilde{\gamma}_{x_{1}}(r)$ is positive definite and since $T<1$ we have that

$$
\tilde{\gamma}_{x_{1}}\left(V_{2 T}\left(x_{2}\right)\right)-\rho\left(V_{2 T}\left(x_{2}\right)\right)=-a_{3 b}\left(V_{2 T}\left(x_{2}\right)\right)=-a_{3 b} \circ \rho^{-1}\left(V_{T}(x)\right) \leq-T a_{3 b} \circ \rho^{-1}\left(V_{T}(x)\right) .
$$

Finally, we consider the third term. Let $\varepsilon_{1} \in \mathcal{K}_{\infty}$ be such that Id $-\varepsilon_{1}$ is a positive definite function. If $\rho\left(V_{2 T}\left(x_{2}\right)\right)>\varepsilon_{1}^{-1} \circ\left(\gamma_{u_{1}}(|u|)+\nu_{1}\right)$ then it holds that

$$
-\rho\left(V_{2 T}\left(x_{2}\right)\right)+\gamma_{u_{1}}(|u|)+\nu_{1} \leq-\left(\operatorname{Id}-\varepsilon_{1}\right) \circ \rho\left(V_{2 T}\left(x_{2}\right)\right) .
$$

Since $V_{T}(x)=\rho\left(V_{2 T}\left(x_{2}\right)\right)$ and using the definition of $\nu_{1}$ and $T<1$, we can write:

$$
\begin{aligned}
V_{T}(x)>\varepsilon_{1}^{-1} \circ \gamma_{u_{1}}(|u|)+\nu & \Rightarrow \rho\left(V_{2 T}\left(x_{2}\right)\right)>\varepsilon_{1}^{-1} \circ\left(\gamma_{u_{1}}(|u|)+\nu_{1}\right) \\
& \Rightarrow-\left(\operatorname{Id}-\varepsilon_{1}\right) \circ \rho\left(V_{2 T}\left(x_{2}\right)\right)=-\left(\operatorname{Id}-\varepsilon_{1}\right)\left(V_{T}(x)\right) \\
& =:-a_{3 c}\left(V_{T}(x)\right) \leq-T a_{3 c}\left(V_{T}(x)\right) .
\end{aligned}
$$


Combining inequalities (35), (39) and (41), with $a_{3}(r):=\min \left\{a_{3 a}(r), a_{3 b} \circ \rho^{-1}(r), a_{3 c}(r)\right\}$, we have that

$$
V_{T}(x)>\varepsilon_{1}^{-1} \circ \gamma_{u_{1}}(|u|)+\nu \Rightarrow \Delta V_{T} \leq-T a_{3}\left(V_{T}(x)\right) .
$$

Case 4: $V_{1 T}\left(x_{1}\right) \geq \rho\left(V_{2 T}\left(x_{2}\right)\right)$ and $V_{1 T}\left(F_{1 T}\right)<\rho\left(V_{2 T}\left(F_{2 T}\right)\right)$. Using condition (27) we have

$$
\begin{aligned}
\Delta V_{T} & =\rho\left(V_{2 T}\left(F_{2 T}\right)\right)-V_{1 T}\left(x_{1}\right) \\
& \leq \max \left\{\rho \circ\left(\mathrm{Id}-T \alpha_{2}\right)\left(V_{2 T}\left(x_{2}\right)\right), \rho \circ \tilde{\gamma}_{x_{2}}\left(V_{1 T}\left(x_{1}\right)\right), \rho\left(\gamma_{u_{2}}(|u|)+\nu_{2}\right)\right\}-V_{1 T}\left(x_{1}\right) .
\end{aligned}
$$

Now we bound the terms on the right hand side of (43). First, using (62) of Lemma 6.3 with $s=V_{2 T}\left(x_{2}\right)$ and $r=V_{1 T}\left(x_{1}\right)$ we can write

$$
\rho \circ\left(\operatorname{Id}-T \alpha_{2}\right)\left(V_{2 T}\left(x_{2}\right)\right)-V_{1 T}\left(x_{1}\right) \leq-T a_{4 a}\left(V_{1 T}\left(x_{1}\right)\right)=-T a_{4 a}\left(V_{T}(x)\right) .
$$

Since $a_{4 b}(r):=\left(\operatorname{Id}-\rho \circ \tilde{\gamma}_{x_{2}}\right)(r)$ is positive definite, $T<1$ and $V_{T}(x)=V_{1 T}\left(x_{1}\right)$, we have that

$$
\begin{aligned}
\rho \circ \tilde{\gamma}_{x_{2}}\left(V_{1 T}\left(x_{1}\right)\right)-V_{1 T}\left(x_{1}\right) & \leq\left(\rho \circ \tilde{\gamma}_{x_{2}}-\mathrm{Id}\right)\left(V_{1 T}\left(x_{1}\right)\right) \\
& =:-a_{4 b}\left(V_{1 T}\left(x_{1}\right)\right)=-a_{4 b}\left(V_{T}(x)\right) \leq-T a_{4 b}\left(V_{T}(x)\right) .
\end{aligned}
$$

Finally, we consider the third term. Let $\varepsilon_{2} \in \mathcal{K}_{\infty}$ is such that $a_{4 c}:=\operatorname{Id}-\varepsilon_{2}$ is a positive definite function. Using the definition of $\nu_{2}, V_{T}(x)=V_{1 T}\left(x_{1}\right)$ and the fact that $T<1$, we can write that

$$
\begin{aligned}
V_{T}(x) \geq \varepsilon_{2}^{-1} \circ \rho \circ \gamma_{u_{2}}(|u|)+\nu & \Rightarrow V_{1 T}(x) \geq \varepsilon_{2}^{-1} \circ \rho\left(\gamma_{u_{2}}(|u|)+\nu_{2}\right) \\
& \Rightarrow-\left(\operatorname{Id}-\varepsilon_{2}\right)\left(V_{T}(x)\right)=:-a_{4 c}\left(V_{T}(x)\right) \leq-T a_{4 c}\left(V_{T}(x)\right) .
\end{aligned}
$$

Combining (44), (45) and (46), with $a_{4}(r):=\min \left\{a_{4 a}(r), a_{4 b}(r), a_{4 c}(r)\right\}$, we can write that

$$
V_{T}(x) \geq \varepsilon_{2}^{-1} \circ \rho \circ \gamma_{u_{2}}(|u|)+\nu \Rightarrow \Delta V_{T} \leq-T a_{4}\left(V_{T}(x)\right) .
$$

By combining (31), (36), (42) and (47) and the fact that $\varepsilon_{i}^{-1}(r)>r, \forall r>0, i=1,2$, we have shown that (3) holds with

$$
\begin{aligned}
& \alpha(r):=\min \left\{\alpha_{1}(r), a_{2}(r), a_{3}(r), a_{4}(r)\right\} \\
& \gamma(r):=\max \left\{\varepsilon_{1}^{-1} \circ \gamma_{u_{1}}(r), \varepsilon_{2}^{-1} \circ \rho \circ \gamma_{u_{2}}(r)\right\}
\end{aligned}
$$

where $\alpha$ is a positive definite function and and $\gamma \in \mathcal{K}$. Hence, the system (11) is SP-ISS.

The last thing left to prove is that if $V_{1 T}$ and $V_{2 T}$ are Lipschitz, uniformly in small $T$ then $V_{T}$ is Lipschitz, uniformly in small $T$. Let $\Delta_{x}>0$ be given. Let $L_{1}, T_{1}^{*}$ and $L_{2}, T_{2}^{*}$ come respectively from the Lipschitz properties of $V_{1 T}$ and $V_{2 T}$ for the set $\left|x_{i}\right| \leq \Delta_{x}, i=1,2$. Note also that since $\rho \in C^{1}$, it is locally Lipschitz and let $L_{\rho}$ be its Lipschitz constant for the set $V_{2 T}\left(x_{2}\right) \leq \bar{\alpha}_{2}\left(\Delta_{x}\right)$. Denote $x:=\left(x_{1}^{T} x_{2}^{T}\right)^{T}$ and $y:=\left(\begin{array}{ll}y_{1}^{T} & y_{2}^{T}\end{array}\right)^{T}$. Let $T^{*}:=\min \left\{1, T_{1}^{*}, T_{2}^{*}\right\}$ and consider arbitrary $T \in\left(0, T^{*}\right)$ and $\max \{|x|,|y|\} \leq \Delta_{x}$. Introduce the sets: $A:=\left\{x: V_{1 T}\left(x_{1}\right)>\rho\left(V_{2 T}\left(x_{2}\right)\right)\right\} ; B:=\left\{x: V_{1 T}\left(x_{1}\right)=\rho\left(V_{2 T}\left(x_{2}\right)\right)\right\} ; C:=\{x:$ $\left.V_{1 T}\left(x_{1}\right)<\rho\left(V_{2 T}\left(x_{2}\right)\right)\right\}$. We consider the following cases, to prove our claim:

Case 1: $(x, y \in A)$ or $(x \in A$ and $y \in B)$ or $(x \in B$ and $y \in A)$ or $(x, y \in B)$

$$
\left|V_{T}(x)-V_{T}(y)\right|=\left|V_{1 T}\left(x_{1}\right)-V_{1 T}\left(y_{1}\right)\right| \leq L_{1}\left|x_{1}-y_{1}\right| .
$$


Case 2: $(x, y \in C)$ or $(x \in C$ and $y \in B)$ or $(x \in B$ and $y \in C)$.

$$
\left|V_{T}(x)-V_{T}(y)\right|=\left|\rho\left(V_{2 T}\left(x_{2}\right)\right)-\rho\left(V_{2 T}\left(y_{2}\right)\right)\right| \leq L_{\rho} L_{2}\left|x_{2}-y_{2}\right| .
$$

Case 3: $x \in A$ and $y \in C$

$$
\left|V_{T}(x)-V_{T}(y)\right|=\left|V_{1 T}\left(x_{1}\right)-\rho\left(V_{2 T}\left(y_{2}\right)\right)\right| .
$$

Since $x \in A$ implies $V_{1 T}\left(x_{1}\right)>\rho\left(V_{2 T}\left(x_{2}\right)\right)$ and $y \in C$ implies $V_{1 T}\left(y_{1}\right)<\rho\left(V_{2 T}\left(y_{2}\right)\right)$, we have that:

1. If $V_{1 T}\left(x_{1}\right)>\rho\left(V_{2 T}\left(y_{2}\right)\right)$ then

$$
\left|V_{1 T}\left(x_{1}\right)-\rho\left(V_{2 T}\left(y_{2}\right)\right)\right|=V_{1 T}\left(x_{1}\right)-\rho\left(V_{2 T}\left(y_{2}\right)\right) \leq V_{1 T}\left(x_{1}\right)-V_{1 T}\left(y_{1}\right) \leq L_{1}\left|x_{1}-y_{1}\right| .
$$

2. If $V_{1 T}\left(x_{1}\right) \leq \rho\left(V_{2 T}\left(y_{2}\right)\right)$ then

$$
\left|V_{1 T}\left(x_{1}\right)-\rho\left(V_{2 T}\left(y_{2}\right)\right)\right|=-V_{1 T}\left(x_{1}\right)+\rho\left(V_{2 T}\left(y_{2}\right)\right) \leq \rho\left(V_{2 T}\left(y_{2}\right)\right)-\rho\left(V_{2 T}\left(x_{2}\right)\right) \leq L_{\rho} L_{2}\left|x_{2}-y_{2}\right| .
$$

Case 4: $x \in C$ and $y \in A$. This case follows by symmetry from Case 3 .

Hence, we can conclude that

$$
\left|V_{T}(x)-V_{T}(y)\right| \leq L\left(\left|x_{1}-y_{1}\right|+\left|x_{2}-y_{2}\right|\right),
$$

where $L:=\max \left\{L_{1}, L_{\rho} L_{2}\right\}$. Therefore, $V_{T}$ is Lipschitz uniformly in small $T$.

Remark 3.1 Similar results can be stated for non-parameterized discrete-time systems $x(k+1)=$ $F(x(k), u(k))$, if all conditions hold on appropriate sets. The relationship between these sets can be easily deduced from the proof of Theorem 3.1. Moreover, similar results can also be presented for another class of parameterized systems $x(k+1)=F_{T, h}(x(k), u(k))$, which naturally arise when a family of approximate discrete-time models of the continuous-time plant is generated by integrating continuoustime plant dynamics over one sampling interval of length $T>0$ using a numerical integration scheme with integration period $h>0$. In particular, the results that we stated can be regarded as a special case of this more general situation when $T=h$ (see [8] for more details).

Remark 3.2 We note that the proofs of the continuous-time result in [4] and the discrete-time result in Theorem 3.1 are notably different although the constructed function $V_{T}$ has the same form. In particular, while the result in [4] was proved by considering 3 different cases, we need to consider 4 cases in discretetime, some of which contained up to three different sub-cases. Moreover, in the proof of the discrete-time result we needed to use The Mean Value Theorem and Lemma 6.2, which were not needed in the proof of the continuous-time result in [4].

\section{Example}

The following example illustrates that it may happen that an approximate discrete-time model satisfies a small gain condition but if the gains depend on $T$ (hence, the subsystems are not SP-ISS in the sense of our Definition 2.1), then the approximate discrete-time model may be stable for all small values of $T$ but the exact discrete-time model is unstable for all small values of $T$. This example motivates our 
approach and in particular the consideration of families of parameterized discrete-time systems and the SP-ISS property that we use.

Consider a continuous-time plant $\dot{x}_{1}=x_{1}+u$, which is between a sampler and zero order hold. Suppose that we want to carry out the controller design using the Euler discrete-time approximate model of the plant

$$
x_{1}(k+1)=(1+T) x_{1}(k)+T u(k) .
$$

Suppose that we use the following family of dynamic controllers

$$
\begin{aligned}
x_{2}(k+1) & =-0.5 x_{2}(k)-T^{2} x_{1}(k), \\
u(k) & =-\frac{1}{T} x_{2}(k)-\frac{2}{T} x_{1}(k) .
\end{aligned}
$$

Note that the approximate closed-loop system $(56),(57),(58)$ can be regarded as a feedback interconnection of two scalar systems (56) with (58) and (57). Moreover, using Lyapunov functions $V_{1 T}\left(x_{1}\right)=\left|x_{1}\right|$ and $V_{2 T}\left(x_{2}\right)=\left|x_{2}\right|$ and suppose that $T<1$, we can write the following:

$$
\begin{gathered}
\left|x_{1}\right| \geq \frac{2}{T}\left|x_{2}\right| \Rightarrow \Delta V_{1 T} \leq-\frac{T}{2}\left|x_{1}\right|, \\
\left|x_{2}\right| \geq 4 T^{2}\left|x_{1}\right| \Rightarrow \Delta V_{2 T} \leq-\frac{T}{4}\left|x_{2}\right| .
\end{gathered}
$$

In this case the gains are $\gamma_{x_{1}}(s)=\frac{2}{T} s$ and $\gamma_{x_{2}}(s)=4 T^{2} s$. Note that for any $M \in(0,1 / 8)$ there exist sufficiently small $\tau_{1}, \tau_{2} \in \mathcal{K}_{\infty}$ so that our small gain condition holds for all $T \in(0, M]$. We have computed the eigenvalues of the approximate closed-loop system matrix and obtained that $\lambda_{1}^{a}=-\frac{1}{2}+2 T^{2}+O\left(T^{3}\right)$ and $\lambda_{2}^{a}=-1+T-2 T^{2}+O\left(T^{3}\right)$, which indicates that indeed the approximate closed-loop model is stable for sufficiently small $T$. However, if we consider the exact closed-loop system consisting of the exact discrete-time plant model $x_{1}(k+1)=e^{T} x_{1}(k)+\left(e^{T}-1\right) u(k)$ and $(57)$, (58), we obtain that the eigenvalues of the system matrix are $\lambda_{1}^{e}=-\frac{1}{2}+2 T^{2}+O\left(T^{3}\right)$ and $\lambda_{2}^{e}=-1-\frac{11}{6} T^{2}+O\left(T^{3}\right)$ and obviously we have that $\left|\lambda_{2}^{e}\right|>1$ for all sufficiently small $T$. In this case, since $\gamma_{x_{1}}$ and $\gamma_{x_{2}}$ depend on $T$, it is not possible to construct a Lyapunov function $V_{T}$ via (15) that satisfies appropriate bounds in Definition 2.1 uniformly in small $T$.

\section{Conclusions}

We have presented in this paper a Lyapunov based small gain theorem for parameterized discretetime SP-ISS systems. This is a discrete-time counterpart of the continuous-time results in [4]. We have presented an example that motivates our results in the case when a discrete-time controller for a sampled-data plant is based on its approximate discrete-time model.

\section{References}

[1] D. Angeli, E. D. Sontag and Y. Wang, "A characterization of integral input to state stability," IEEE Trans. Automat. Contr., vol.45 no. 6 pp. 1082-1097, 2000.

[2] B. Ingalls and E. D. Sontag, "A small-gain theorem with applications to input/output systems, incremental stability, detectability, and interconnections," J. of The Franklin Institute, vol. 339, pp. 211-229, 2002. 
[3] Z. P. Jiang, A. R. Teel and L. Praly, "Small-gain theorem for ISS systems and applications," Math. Control Signals Systems, vol. 7, pp 95-120, 1994.

[4] Z. P. Jiang, I. M. Y. Mareels and Y. Wang, "A Lyapunov formulation of the nonlinear small-gain theorem for interconnected ISS systems," Automatica, vol. 32, no. 8, pp. 1211-1215, 1996.

[5] Z. P. Jiang and Y. Wang, "Input-to-state stability for discrete-time nonlinear systems," Automatica, vol. 37, pp. 857-869, 2001.

[6] D. S. Laila and D. Nešić, "Changing supply rates for input-output to state stable discrete-time nonlinear systems with applications", to appear in Automatica, June 2003.

[7] D. Nešić, A. R. Teel and P. Kokotović, "Sufficient conditions for stabilization of sampled-data nonlinear systems via discrete-time approximations," Syst. Contr. Lett., vol. 38, pp. 259-270, 1999.

[8] D. Nešić and A. R. Teel, "A framework for stabilization of nonlinear sampled-data systems based on their approximate discrete-time models", accepted subject to minor revision in IEEE Trans. Automat. Contr., 2002.

[9] D. Nešić and D. S. Laila, "A note on input-to-state stabilization for nonlinear sampled-data systems," IEEE Trans. Automat. Contr., vol. 47, pp. 1153-1158, 2002.

[10] D. Nešić and A.R. Teel, "Changing supply functions in input-to-state stable systems: the discretetime case", IEEE Trans. Automat. Contr., vol. 46, pp. 960-962, 2001.

[11] L. Praly and Y. Wang, "Stabilization in spite of matched unmodeled dynamics and an equivalent definition of input to state stability," Math. Control Signals Systems, vol. 9, pp. 1-33, 1996.

[12] E. D. Sontag "Smooth stabilization implies coprime factorization," IEEE Trans. Automatic Control, vol. 34, pp. 435-443, 1989.

[13] E. D. Sontag, "The ISS philosophy as a unifying framework for stability-like behavior," in Nonlinear Control in the Year 2000 (Volume 2) (Lecture Notes in Control and Information Sciences, A. Isidori, F. Lamnabhi-Lagarrigue, and W. Respondek, eds.), Springer, Berlin, 2000, pp. 443-468.

[14] A. R. Teel, "A nonlinear small gain theorem for the analysis of control systems with saturation," IEEE Trans. Automat. Contr., vol. 41, pp. 1256-1270, 1996.

[15] M. P. Tzamtzi and S. G. Tzafestas, "A small gain theorem for locally input to state stable interconnected systems," J. of The Franklin Institute, vol. 336, pp. 893-901, 1999.

\section{Appendix}

The following technical lemmas are used to prove the main result. 
Lemma 6.1 [4] Let $\sigma_{1} \in \mathcal{K}$ and $\sigma_{2} \in \mathcal{K}_{\infty}$ satisfy $\sigma_{1}(r)<\sigma_{2}(r)$ for all $r>0$. Then there exists a $\mathcal{K}_{\infty}$ function $\sigma$ such that

- $\sigma_{1}(r)<\sigma(r)<\sigma_{2}(r)$ for all $r>0$;

- $\sigma(r)$ is $C^{1}$ on $(0, \infty)$ and $\sigma^{\prime}(r)=: \tilde{q}(r)$ is a positive function.

Note that the above given function $\tilde{q}$ is positive but it is not positive definite in general. The following lemma is a simple consequence of [1, Lemma IV.1]

Lemma 6.2 [1] Let $\tilde{q}: \mathbb{R}_{\geq 0} \rightarrow \mathbb{R}_{>0}$ be a positive function. Then there exist a positive definite function $q$ and functions $q_{1} \in \mathcal{K}_{\infty}$ and $q_{2} \in \mathcal{L}$ such that $\tilde{q}(r) \geq q(r) \geq q_{1}(r) \cdot q_{2}(r), \forall r \geq 0$.

Note that the existence of $q$ is trivial to show, whereas the existence of $q_{1}$ and $q_{2}$ was proved in [1].

Lemma 6.3 Suppose that we are given a function $\rho \in \mathcal{K}_{\infty}$ where $q(r):=\rho^{\prime}(r)$ is a positive function, a positive definite function $\alpha$, such that $(\operatorname{Id}-T \alpha)$ is positive definite and $T \in[0,1)$. Then, we can write:

$$
\begin{gathered}
\max _{0 \leq s \leq \rho(r)}(\operatorname{Id}-T \alpha)(s)-\rho(r) \leq-T a_{1} \circ \rho(r) \\
\max _{0 \leq \rho(s) \leq r} \rho \circ(\operatorname{Id}-T \alpha)(s)-r \leq-T a_{2}(r),
\end{gathered}
$$

for some positive definite functions $a_{1}$ and $a_{2}$.

Proof of Lemma 6.3: The inequality (61) follows easily from considering two cases $s \leq \frac{\rho(r)}{2}$ and $\frac{\rho(r)}{2} \leq s \leq \rho(r)$. In particular, we obtain $a_{1}(r):=\max \left\{\frac{1}{2} r, \alpha(r)\right\}$. We now prove (62) in more detail. First, note that if $0 \leq \rho(s) \leq \frac{r}{2}$, then

$$
\rho \circ(I d-T \alpha)(s)-r \leq \rho(s)-r \leq-\frac{r}{2} .
$$

Consider now $\rho(s) \in\left[\frac{r}{2}, r\right]$. First, we use the Mean Value Theorem to write:

$$
\rho \circ(I d-T \alpha)(s)-r \leq \max _{\frac{r}{2} \leq \rho(s) \leq r} \rho \circ(I d-T \alpha)(s)-\rho(s)=q\left(s^{*}\right)[-T \alpha(s)],
$$

where $s^{*} \in[(I d-\alpha)(s), s]$ since $T<1$. Using Lemma 6.2 we can find two functions $q_{1} \in \mathcal{K}_{\infty}$ and $q_{2} \in \mathcal{L}$ such that

$$
-T q\left(s^{*}\right) \alpha(s) \leq-T q_{1}\left(s^{*}\right) \cdot q_{2}\left(s^{*}\right) \cdot \alpha(s) \leq-T q_{1} \circ(I d-\alpha)(s) \cdot q_{2}(s) \cdot \alpha(s)=:-T \alpha^{*}(s),
$$

where $\alpha^{*}(\cdot)$ is a positive definite function. Applying Lemma 6.2 again we obtain $q_{1}^{*} \in \mathcal{K}_{\infty}, q_{2}^{*} \in \mathcal{L}$ and then using the fact that $s \in\left[\rho^{-1}(r / 2), \rho^{-1}(r)\right]$ we can write:

$$
-T \alpha^{*}(s) \leq-T q_{1}^{*}(s) \cdot q_{2}^{*}(s) \leq-T q_{1}^{*} \circ \rho^{-1}(r / 2) \cdot q_{2}^{*} \circ \rho^{-1}(r)=:-T \alpha_{1}(r) .
$$

This completes the proof of (62) with $a_{2}(r):=\min \left\{r / 2, \alpha_{1}(r)\right\}$. 